\title{
Acquisition et structure des schémas sociolinguistiques en langue étrangère
}

\author{
Gabriela Viana dos Santos ${ }^{1, *}$, Laurence Buson ${ }^{2}$, et Jean-Pierre Chevrot ${ }^{3}$ \\ ${ }^{1}$ Université Grenoble Alpes, CS 4070038058 Grenoble cedex 9, France \\ ${ }^{2}$ Université Grenoble Alpes, CS 4070038058 Grenoble cedex 9, France \\ ${ }^{3}$ Université Grenoble Alpes, CS 4070038058 Grenoble cedex 9, France
}

\begin{abstract}
Résumé. L'une des particularités du processus d'acquisition sociolinguistique en langue étrangère porte sur la difficulté des apprenants à intégrer les normes sociolinguistiques de la langue cible. Si dans leur culture native, les apprenants construisent un réseau d'associations entre connaissances linguistiques et connaissances sociales, en langue étrangère ils doivent transposer cette capacité. La question importante est de savoir comment un apprenant développe cette compétence en langue étrangère s'il n'a pas les mêmes repères sociaux que les natifs. Les études en langues étrangères montrent que les apprenants utilisent davantage les variantes sociolinguistiques formelles si comparés aux natifs. Quelques travaux se basent sur la théorie des schémas pour expliquer cette différence. Ces études indiquent que les apprenants possèdent des schémas incomplets en langue cible. Dans cet article, nous avons mené une étude auprès de 66 étudiants de FLE, 24 anglophones et 42 sinophones à partir du Test de Répétion Sociolinguistique. Les résultats montrent que, tel que les natifs, les étudiants modifient les variantes sociolinguistiques non compatibles avec l'énoncé en le rendant homogène. Nous mettons ainsi en évidence l'existence des schémas sociolinguistiques complets chez les apprenants de langues étrangères. En outre, notre étude montre une différence dans la construction des schémas en fonction du pays d'origine des apprenants.
\end{abstract}

\begin{abstract}
Foreign language sociolinguistic schemata acquisition and structure. One of the particularity of the process of sociolinguistic acquisition in a foreign language concerns the difficulty of leaner to integrate the sociolinguistic norms of the target language. If in their native culture, the learners build a network of associations between linguistic knowledge and social knowledge, in foreign language they must transpose this capacity. The important question is how a learner develops this competence in a foreign language if he does not have the same social references as the native ones. Studies in foreign languages show that learners make more use of formal sociolinguistic variants when compared to native speakers. Works based on schema theories advocate that learners have incomplete schemas in the target language as to explain this difference. In this work we conducted a study of 66 students of French language, 24 of them being native speakers of English and 42 being native
\end{abstract}

* Corresponding author : s.vianagabriela@gmail.com 
speakers of Chinese. The experiment is based on the Sociolinguistic Repetition Task. The results show that, like natives, students modify sociolinguistic variants that are not compatible with the statement by making it homogeneous. We thus highlight the existence of complete sociolinguistic schemata among learners of foreign languages. We also show differences in the schemata structure according to the native country of the students.

\section{Introduction}

Le Cadre Européen Commun de Référence pour les Langues (CECRL, 2001) établie que l'enseignement d'une langue seconde doit porter sur les compétences linguistiques, sociolinguistiques et pragmatiques. Ces objectifs impliquent un apprentissage au-delà des simples règles grammaticales : les apprenants doivent acquérir une flexibilité sociolinguistique permettant des échanges en langue cible dans des contextes divers.

La compétence sociolinguistique est définie comme « la capacité à reconnaître et à produire un langage socialement adapté selon le contexte »(Lyster, 1994: 263). L'acquisition de cette compétence a fait l'objet de plusieurs études dans le domaine des langues étrangères. La particularité du processus de l'acquisition sociolinguistique vient de la difficulté des apprenants à intégrer et assimiler les normes sociolinguistiques en langue cible. En effet, comme l'affirme Dewaele \& Wourm (2001:130), il est beaucoup plus simple d'expliquer aux étudiants des règles grammaticales « que de faire comprendre que certains mots, expressions, constructions syntaxiques ne sont appropriés que dans certains contextes spécifiques », notamment par les « nuances que ceux-ci transmettent et les innombrables facteurs dans la situation qui déterminent leur usage $»$.

Alors que les travaux en langue maternelle montrent que les natifs maitrisent les variations sociolinguistiques de leur langue et qu'ils peuvent s'adapter aux différentes situations de communication dès l'enfance (Nardy et al., 2013) en langue étrangère, l'apparition de telles capacités est beaucoup plus lente. S'il ne fait pas de doute que dans leur culture native, ils construisent un réseau d'associations entre connaissances sociales et connaissances linguistiques, en langue étrangère un tel faisceau d'associations doit être mis en place. La question importante est de savoir comment un apprenant développe cette compétence en langue étrangère s'il n'a pas les mêmes repères sociaux que les natifs.

Plusieurs études montrent que les apprenants utilisent davantage les variantes sociolinguistiques formelles si comparés aux natifs dans différentes situations de communication (Regan, 1995, 1996, 1997 ; Regan \& Dewaele, 2002 ; Mougeon et al., 2002 ; Howard, 2012 ; Van Compernolle, 2013). Ces études mettent l'accent sur écart entre la production des natifs et non natifs et expliquent que plusieurs facteurs, comme le contexte syntaxique des phrases (Howard, 2012) et l'input institutionnel, (Mougeon et al.,2002) influencent la production des apprenants.

D'autres travaux (Ranney, 1992; Dewaele, 2002; Dewaele \& Wourm, 2002) s'appuient sur la théorie des schémas (Anderson, 1997 ; Rumelhart, 1980) pour expliquer les différences sociolinguistiques entre natifs et non natifs. Un schéma est défini comme un ensemble structuré de connaissances. Il regroupe, en plus des connaissances elles-mêmes, la façon d'employer ces connaissances, des situations, des événements, des actions et des séquences d'actions. Dans ce sens, le schéma sociolinguistique représente les relations établies entre ces différentes composantes, portant notamment sur le savoir social et linguistique.

En se basant sur la théorie des schémas, Ranney (1992) examine la perception des demandes sociolinguistiques liées à une consultation médicale au États-Unis chez les 
étudiants des langues étrangères. L'auteure affirme que les schémas sont importants dans la compréhension des tâches et événements, car ils permettent d'inférer et de comprendre l'intention communicationnelle du discours dans un contexte spécifique. Les résultats de son étude montrent que malgré une notion générale des schémas discursifs, les schémas représentant différents tâches et actions accomplies lors d'une activité sont moins établis ou non-cohérents, si comparés aux natifs. En outre, leurs actes de langage sont plus formels aussi bien que leur rapport avec les médecins.

De la même manière, Dewaele (2002) puis Dewaele \& Wourm (2002) soutiennent que les apprenants des langues étrangères ont des schémas incomplets en langue cible. Les auteurs analysent l'usage de pronoms d'allocution $t u$ et vous chez les apprenants de FLE et chez des francophones natifs. Les résultats montrent que l'usage du pronom tu est significativement moins importante chez les apprenants. De plus, ces derniers alternent l'usage des deux pronoms dans le même contexte de communication. D'après les auteurs, la raison du «flottement » dans l'usage des pronoms vient des lacunes existantes dans les schémas établis en langue étrangère.

Cependant, d'autres travaux indiquent qu'en langue étrangère les apprenants se basent dans leurs schémas construits en langue maternelle et qu'ils les modifient au fur et à mesure $\mathrm{du}$ processus d'acquisition. Selon Kasper (2001, p.511) les études sur l'acquisition de compétences pragmatiques montrent que les apprenants s'appuient « systématiquement sur les connaissances pragmatiques universelles et celles de leur langue maternelle ».

En outre, les schémas que les apprenants des langues étrangères apportent de leur culture peuvent influencer l'exécution des tâches en langue cible. Par exemple, les études sur l'acquisition des compétences en lecture indiquent que les schémas liés aux savoirs et aux croyances culturelles des apprenants (schémas de contenu) ont un effet plus important que la complexité syntaxique (Johnson, 1981) ou l'organisation rhétorique du texte (Carrell, 1990) lors des tâches de lecture. Dans cette perspective, il se peut que les schémas de contenu d'origine des apprenants puissent amener les étudiants à percevoir les différences sociales du langage d'une façon différente de celle des natifs.

En langue maternelle, deux études montrent que les natifs construisent des schémas sociolinguistiques cohérents (Buson, 2009) et (Buson et al., in press) D'après ces études, lors d'un test de répétition, les natifs ont tendance à remplacer une variante sociolinguistique non compatible avec les autres variables sociolinguistiques de l'énoncé, en le rendant homogène. Par exemple, lors de l'écoute d'un énoncé formel non homogène (lexique et contenu du message formels plus une variante non standard), les sujets répètent l'énoncé en remplaçant la variante non standard par une variante standard. Ces études mettent en évidence l'existence des schémas cohérents qui influencent la répétition des énoncés.

$\mathrm{Vu}$ que la compétence sociolinguistique dépend de l'agencement de connaissances sociales et linguistiques, le développement des schémas sociolinguistiques en langue étrangère pourrait être une étape importante dans le processus d'acquisition. Dans cette perspective, il est important de poser deux questions préalables :

1. Comment sont organisées cognitivement les variantes sociolinguistiques chez les apprenants ? Sont-elles regroupées de façon cohérente, selon leur valeur indexicale? En d'autres mots, est-ce que les étudiants regroupent les variantes standard de différents niveaux linguistiques mais de même valeur indexicale? (par exemple le «ne » de négation avec la liaison réalisée et l'interrogation par inversion)

2. Est-ce que l'organisation cognitive des variantes sociolinguistiques dépend de la langue ou de la culture d'origine ? Cette seconde question est un premier pas pour explorer la possibilité que des apprenants des langues et des cultures différentes se basent sur des schémas sociaux et culturels acquis antérieurement pour construire leur compétence sociolinguistique en L2. 


\section{Méthodologie et Prédictions}

Pour comprendre la structure du schéma sociolinguistique en LE, nous avons mené une étude auprès de 66 apprenants de FLE en séjour d'étude en France. Le groupe d'étudiants est formé par 24 anglophones ( 8 hommes et 16 femmes, âgés en moyenne de 21,6 ans), dont 7 sont d'origine britannique, 16 sont d'origine américaine et 1 est d'origine australienne, et par 42 étudiants sinophones ( 21 hommes et 21 femmes, âgés en moyenne de 24,4 ans), dont 41 sont d'origine chinoise et 1 est d'origine taiwanaise. Les étudiants anglophones ont en moyenne 8 ans d'étude de la langue française et leur temps moyen de séjour en France est de 2,8 ans. Les étudiants sinophones ont en moyenne 2,8 ans d'études de la langue française et leur temps moyen de séjour en France est de 2,6 ans. La plupart des étudiants des deux groupes ont un niveau B2 en langue française.

Pour réaliser cette étude, nous avons utilisé le Test de Répétition Sociolinguistique (Buson, 2009 ; Buson et al., in press), en respectant toutes les étapes du protocole. Cette tâche consiste à écouter et répéter des énoncés formels et informels, homogènes ou non homogènes du point de vue sociolinguistique. Un énoncé formel présente un contenu et un lexique formel, des constructions grammaticales standard et tous les traits phonologiques standard ont été maintenus. Inversement, un énonce informel présente un contenu et un lexique informel, des constructions grammaticales non standard et des traits phonologiques non standard. Dans les énonces formels homogènes, toutes les variantes sont en accord avec le reste de l'énonce. Par exemple, les liaisons et la particule négative "ne" de négation sont maintenues. Dans les énonces formels non homogènes, une seule variable cible standard contredit le reste de l'énoncé. Par exemple, le "ne" de négation est absent dans un énoncé comportant plusieurs traits standard.

Tableau 1. - Variables sociolinguistiques utilisées dans la tâche de répétition.

\begin{tabular}{|c|c|}
\hline $\begin{array}{c}\text { Variables morpho- } \\
\text { syntaxiques }\end{array}$ & $\begin{array}{c}\text { «ne » de négation - réalisé/ non réalisé. } \\
\text { Pronom relatif dont /que - choix des pronoms. }\end{array}$ \\
\hline $\begin{array}{c}\text { Variable } \\
\text { phonétiques }\end{array}$ & $\begin{array}{c}\text { Liaison facultative }- \text { réalisé/ non réalisé } \\
\text { /1/ dans le pronom clitique « il »- réalisé/ non réalisé }\end{array}$ \\
\hline $\begin{array}{c}\text { Variables } \\
\text { discursives }\end{array}$ & $\begin{array}{c}\text { Particules considérées comme formelles, ex : } \\
\text { euh non, oui bon, }\end{array}$ \\
Particules considérées comme informelles, ex : oh mais, hein
\end{tabular}

Le test est composé par 24 énoncés (Annexes) dont 6 énoncés formels homogènes $(\mathrm{FH})$, 6 énoncés formels non-homogènes $(\mathrm{FnH}), 6$ énoncés informels homogènes $(\mathrm{IH})$ et 6 informels non homogènes ( $\mathrm{InH})$. Dans chaque groupe de six énoncés, les variables sociolinguistiques qui déterminent si l'énoncé est homogène ou non homogène appartiennent à trois niveaux linguistiques différents : deux variables morphosyntaxiques, deux variables phonologiques et deux variables discursives. L'ensemble de variables est détaillé dans le tableau 1.

Pour réaliser ce test, nous avons utilisé le logiciel E-prime. Les 24 énoncés ont été présentés de façon aléatoire et la tâche a eu une durée d'environ 7 minutes. Entre le moment de l'audition de l'énoncé et le moment de la répétition, il a été demandé aux étudiants de lire un nombre affiché sur l'écran de l'ordinateur. Cette étape impose un délai de répétition d'au moins trois secondes entre l'énoncé entendu et la production des apprenants. Ce délai permet de différer la reproduction de l'énoncé, qui doit ainsi être stockée en mémoire de travail avant d'être restituée et éventuellement reconstituée à partir du schéma opérant.

Notre prédiction était que les apprenants en séjour d'études construisent des schémas sociolinguistiques standard et non standard en langue étrangère. En effet, le séjour d'étude, 
donc l'acquisition en milieu naturel, serait favorable à la construction des schémas sociolinguistiques, notamment pour promouvoir des échanges authentiques au pays de la langue cible. Dans ce sens, nous attendons à avoir plus de répétitions non identiques dans les contextes non homogènes que dans les contextes homogènes. Nous prévoyons que, après l'écoute d'un énoncé homogène, les apprenants devraient produire la variante cible de façon identique à l'input. Inversement, lors de l'écoute d'un énoncé non homogène, les apprenants devraient restaurer la variante cible de façon cohérente avec l'ensemble du message, ce qui rend la répétition non identique à l'input. Le tableau 2 illustre cette relation:

Tableau 2. Exemple de production attendue lors de la tâche de répétition dans un contexte non homogène (dans l'input la particule négative «ne » est absente alors que le lexique et le contexte sont formels).

\begin{tabular}{|c|c|}
\hline Input & $\begin{array}{c}\text { C'esT un prix symbolique même si le gouvernement veut pas } \\
\text { parler de gratuité. (sans particule négative «ne »). }\end{array}$ \\
\hline Répétition & $\begin{array}{c}\text { C'esT un prix symbolique même si le gouvernement ne veut pas } \\
\text { parler de gratuité (avec particule négative «ne »). }\end{array}$ \\
\hline
\end{tabular}

En ce qui concerne des différences liées à la langue et à la culture des apprenants, nous voulons examiner, de façon exploratoire, si les répétitions non identiques seront les mêmes pour les anglophones et pour les sinophones. L'apparition de telles différences entre les deux groupes d'apprenants serait un premier indice que des schémas sociolinguistiques hérités de la L1 influencent la compétence sociolinguistique en L2.

\section{Des schémas sociolinguistiques en Langue Étrangère ?}

Cette analyse est centrée sur les variables sociolinguistiques phonologiques et morphosyntaxiques. À ce stade, nous n'avons pas pris en compte les variables discursives pour plusieurs raisons. Tout d'abord, étant donné que l'enseignement des marqueurs discursifs est peu visé en classe de langue étrangère, les apprenants peuvent avoir des schémas concernant l'usage des variantes discursives peut structurés ou inexistantes. En effet, nous avons consulté des sites web des ressources pour les enseignants de FLE, comme le site Le point du FLE et le site TV5.org, ainsi que les méthodes d'enseignent de base, comme La Grammaire Progressive du Français et Communication Progressive du Français, et nous n'avons pas trouvé dans ces références un seul travail explicite sur l'usage des marqueurs discursifs. Dans ce sens, l'apprentissage des ces derniers semble être moins contrôlé que celle des variables morphosyntaxiques et phonologiques. En deuxième lieu, vu la spécificité de ces variables, nous sommes entrain de les traiter séparément.

Pour analyser la structure des schémas sociolinguistiques chez les apprenants, tout d'abord nous nous avons mené une analyse de variance à deux facteurs : homogénéité de l'énoncé (homogène, non-homogène) et formalité de l'énoncé (formel, informel). L'interaction que nous ciblions entre l'homogénéité des énoncés et la formalité des énoncés est significative $(\mathrm{F}(1,32)=46,095, \mathrm{p}<0,0001)$. Les résultats indiquent que les sujets modifient davantage les énoncés non homogènes (en restaurant la variable non compatible avec l'ensemble de l'énoncé) que les énoncés homogènes. Le graphique de la figure 1 illustre cette interaction : 


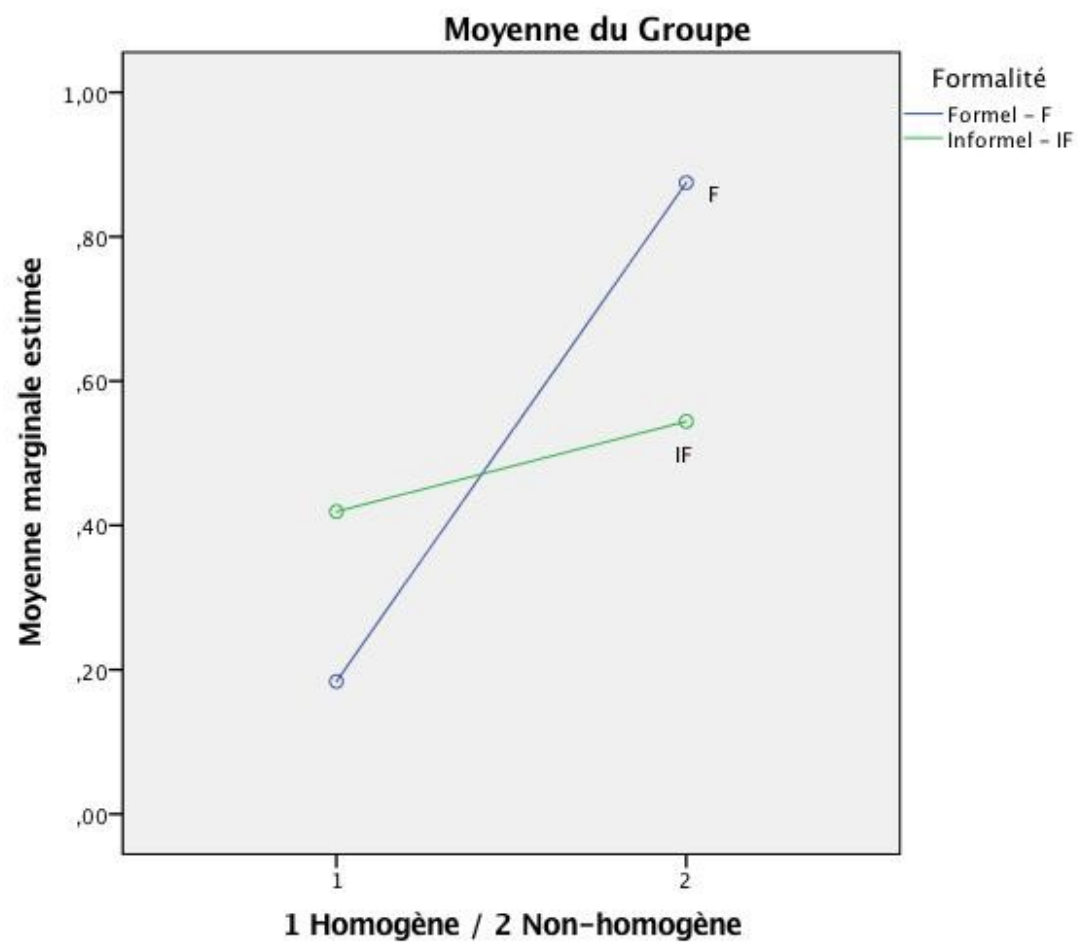

Fig. 1. Interaction entre formalité et hétérogénéité des énoncés.

Les résultats rejoignent notre hypothèse de départ, selon laquelle la construction des schémas sociolinguistiques, c'est à dire, la construction des schémas qui mettent en relation des variantes sociolinguistiques avec leur valeur indexicale, existe chez les apprenants en séjour d'étude. La construction des schémas serait possible grâce à l'acquisition lors échanges authentiques. En effet, plusieurs études montrent que les apprenants ayant plus de contact avec la langue cible sont ceux qui présentent un taux de production de variantes standard le plus bas (Dewaele \& Regan, 2002; Dewaele, 2002; Dewaele, 2004). Les apprenants bénéficiant d'échanges dans des contextes divers ont plus de chance de construire des schémas standard et non standard que les étudiants bénéficiant seulement d'un input formel.

Ces résultats nos permettent également de comparer le sens de la restauration et de savoir si les apprenants passent du sens standard vers le sens non standard, ou inversement du sens non standard vers le sens standard. Autrement dit, nous pouvons savoir si les apprenants ont autant tendance à remplacer les variantes standard par les variantes non standard que les variantes non standard par les variantes standard.

Pour illustrer cette comparaison, prenons le cas de la variable de négation ne (exemple emprunté à Buson et al., 2014) :

1. Formel Homogène : Nous ne connaissions même pas le président $\mathrm{d}(\mathrm{e})$ cette association

2. Informel non Homogène : Ouais bein le gars $i(l)$ n'avait pas franch (e) ment le choix au final

3. Formel non Homogène : CeT un prix symbolique même si le gouvernement veut pas parler de gratuité

4. Informel Homogène : Tu vas pas mettre tes pompes dégueu dans ma caisse, nan? 
Si un ne présent dans l'énoncé informel non homogène est modifié (et donc supprimé) plus que dans l'énoncé formel homogène ; et si, simultanément, un «ne» absent dans un énoncé formel non homogène est modifié (et donc ajouté) plus que dans un énoncé informel homogène, alors nous pouvons conclure que les apprenants modifient les énoncés aussi bien du sens standard vers le non standard que du sens non standard vers le standard.

En comparant FH-InH, nous constatons que les pourcentages moyens des restaurations sont plus importants dans la condition non homogène InH que dans la condition homogène FH. Autrement dit, les apprenants remplacent une variante standard par une variante non standard. Nous trouvons également davantage des modifications dans la condition FnH que dans la condition IH, ce qui signifie que les apprenants remplacent également une variante non standard par une variante standard. Cela montre que les étudiants possèdent un schéma sociolinguistique en langue cible : ils associent les variantes standard avec un contexte formel et les variantes non standard avec un contexte informel. Autrement dit, ils associent les variantes standard et non standard à leur valeur indexicale.

Le taux plus élevé des modifications des énoncés FnH en comparaison avec les énoncés InH met en évidence la tendance des apprenants de modifier les énonces du sens non standard vers le sens standard. Ce résultat peut être expliqué par deux raisons. Tout d'abord par leur apprentissage formel de la langue. En effet, le système standard de la langue est enseigné de façon explicite. Il est bien codifié en classe par les enseignants. Cependant, l'apprentissage du non-standard reste implicite et liée, par exemple, à la vie sociale des étudiants, ce qui explique leur tendance à rendre les énoncés standard.

La différence entre les taux de modifications des énoncés informels nous aide à confirmer notre proposition. Alors que les étudiants modifient davantage les énoncés FnH en comparaison aux énoncés FH (87,5\% contre 18,4\%), le taux de modification entre les énoncés InH et IH est très proche $(54,4 \%$ contre $41,9 \%)$. Ce que montre que les étudiants possèdent un schéma standard formé et un schéma non standard sous-formé ou implicite.

Deuxièmement, le cadre formel de la situation de passation du test a certainement influencé les résultats et a favorisé le remplacement de variantes non standard par des variantes standard. Cette tendance est également présente chez les natifs (Buson et al, in press). Cependant, le taux de modifications des énoncés non homogènes est supérieur à celui des énoncés homogènes.

\subsection{Existe-t-il des schémas sociolinguistiques différents en fonction de la langue et de la culture des apprenants ?}

Pour répondre à la question contenue dans le titre de cette section, nous avons mené une analyse de variance à trois facteurs, à savoir le niveau de formalité (formel/informel) l'homogénéité des énoncés (homogène / non homogène) et selon la nationalité des apprenants (anglophones/ sinophones). Les graphiques de la figure 2 illustrent les résultats de cette interaction $(\mathrm{F}(1,32)=4,11, \mathrm{p}<0,05)$.

Les résultats montrent que la structure du schéma sociolinguistique est différente selon la nationalité des apprenants. Alors que chez les anglophones, nous trouvons une différence entre les taux de modification dans le sens standard et non standard, chez les étudiants sinophones, il n'y a de modifications que dans le sens standard. En effet, la fréquence des modifications dans les conditions homogènes et non homogènes est presque similaire pour les énoncés informels. Ces résultats suggèrent que les étudiants anglophones possèdent des schémas sociolinguistiques standard et non standard en langue étrangère. Inversement, les étudiants sinophones possèdent seulement des schémas sociolinguistiques standard. 


\section{Moyenne - Anglophones}

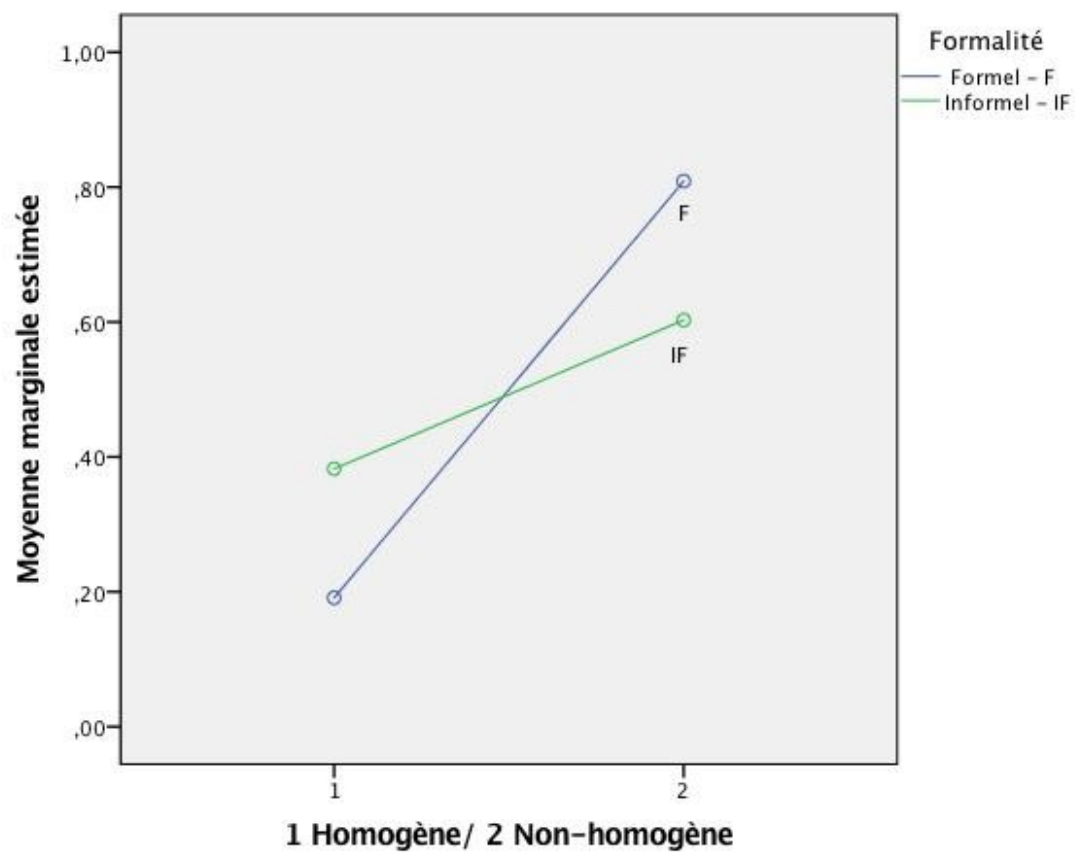

Fig. 2.A. Interaction entre formalité et hétérogénéité des énoncés par nationalité - Anglophones.

\section{Moyenne - Sinophones}

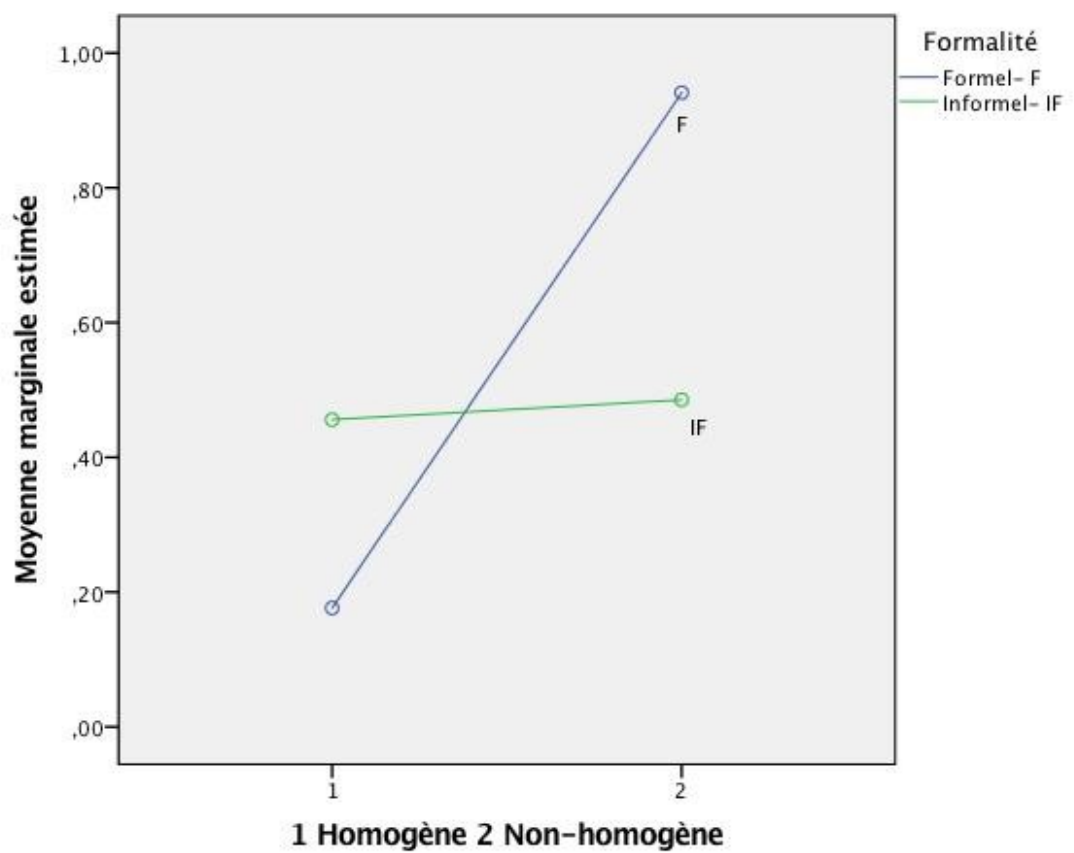

Fig. 2.B. Interaction entre formalité et hétérogénéité des énoncés par nationalité - Sinophones. 


\section{Conclusion et prolongements}

L'apprentissage d'une langue étrangère implique l'acquisition de différents savoirs : le savoir-faire, le savoir-être et le savoir-apprendre. À partir de la mobilisation de ces savoirs, l'apprenant développe diverses compétences qui seront perfectionnées au cours de son apprentissage.

Dans cet article nous avons abordé l'acquisition de compétences sociolinguistiques, avec une question cognitive. À partir du Test de Répétition Sociolinguistique (Buson, 2009 ; Buson et al., in press), nous avons montré que les apprenants des langues étrangères possèdent de schémas sociolinguistiques en langue cible. En effet, tels que les natifs, les apprenants, à l'écoute d'un énoncé hétérogène sur le plan sociolinguistique, modifient la variable non compatible avec l'énoncé afin de le rendre homogène. Ces résultats indiquent que des schémas sociolinguistiques standard et non standard existent en langue cible et qu'ils modifient la répétition des énonces.

Cependant, la structure des schémas diffère selon la langue et l'origine des apprenants. Comme nous l'avons vu, la structure des schémas n'est pas la même au sein du groupe d'étudiants. Alors que les étudiants d'origine américaine possèdent des schémas sociolinguistiques standard et non standard, les étudiants d'origine chinoise présentent seulement des schémas standard.

La relation entre l'organisation des schémas et la langue et culture d'origine peut être expliquée en fonction de différents facteurs. Un premier facteur serait lié au temps d'exposition à la langue française. Bien que les deux groupes d'étudiants possèdent en moyenne le même niveau de langue, le temps d'étude de la langue française entre les deux groupes est très différent. Pour les étudiants sinophones, ce temps est en moyenne de 2,8 ans, alors que pour les anglophones, ce temps s'élève à 8 ans. Cette différence pourrait influencer la construction des schémas des apprenants, les apprenants anglophones ayant bénéficié de plus de temps pour intégrer les usages sociolinguistiques de la langue française pendant leur apprentissage.

Un deuxième facteur serait le type d'enseignement dispensé aux étudiants sinophones et anglophones dans leur pays d'origine. Selon Gautier (2016), l'enseignement des langues en Chine a longtemps priorisé les méthodes traditionnelles. D'après Cuet (2013), les cours de communication et de conversation sont vus comme moins importants par les apprenants. Ce type d'enseignement traditionnel contraste avec méthodes d'enseignement des pays occidentaux, qui valorisent les méthodes communicatives en classe de langue étrangère.

Enfin, un troisième facteur serait que les apprenants importent vers la L2 des schémas socioculturels et des schémas sociolinguistiques qui proviennent de leur langue et de leur culture d'origine. En effet, ces schémas pourraient influencer de façon indirecte l'intégration des connaissances sociolinguistiques dans une autre culture. Notamment, ils pourraient affecter les liens sociaux que les apprenants établissent pendant leur séjour, ou bien la qualité des échanges en langue cible. Par exemple, l'étude de Gautier (2016) sur le réseau social d'étudiants chinois et américains en France met en évidence une relation entre sociabilité et usage de variables sociolinguistiques. Selon cette étude, les étudiants anglophones présentent en majorité des réseaux étendus, caractérisés par un grand nombre de liens et d'individus. Dans ce type de réseau, les étudiants ont tendance à quitter leurs pairs de même nationalité pour établir d'autres types de relations. Inversement, les étudiants chinois présentent des réseaux concentrés, caractérisés par un faible nombre de relations concentrées autour de personnes de même nationalité. Dans ce type de réseau, les étudiants ont très peu des liens avec des francophones. Les résultats indiquent que le fait de créer des réseaux peu cosmopolites et orientés vers des camarades de même nationalité est lié à des usages qui restent plus standard tout au long du séjour. Inversement, les usages des étudiants en contact avec des natifs évoluent vers le non standard. 
Notre étude montre que lors d'un séjour étude, les apprenants construisent de schémas sociolinguistiques. Ils organisent mentalement de façon différente les variables sociolinguistiques standard et non standard en les associant entre elles à l'intérieur de chaque catégorie. Cependant, notre travail nous invite à d'autres questionnements. Les apprenants sont-ils capables de percevoir d'autres dimensions sociales liées aux variables que la seule opposition entre standard et non standard? Quelle est l'origine des différences qui sont apparues entre les schémas construits par des étudiants sinophones et des étudiants anglophones ? Ces différences sont-elles la conséquence de processus d'apprentissages différents ou de transferts de schémas sociolinguistiques et socioculturels différents depuis la langue et la culture d'origine ? Ces questionnements laissent de la place à des futures recherches.

\section{Références bibliographiques}

Anderson, R. C. (1977). The notion of schemata and the educational enterprise : General discussion of conference. In R. C. Anderson, R. J. Spiro, \& W. E. Mon- tague (Eds.), Schooling and the acquisition of knowledge (p. 415-431). Hillsdale, NJ : Lawrence Erlbaum Associates.

Birdsong, D. (2012). Metalinguistic performance and interlinguistic competence, volume 25. Springer Science \& Business Media.

Buson, L. (2009). Stylistic variation and socialisation networks at school : uses, representations, acquisition and educational application with children aged 5 to 11 (Thesis). Université Stendhal Grenoble III.

Buson, L., Chevrot, J. P., Nardy, A., \& Abouzaid, M. (2014). Catégorisation et repré- sentations sociolonguistiques : les variétés stylistiques existent-elles? Congrès Mondial de Linguistique Française - CMLF, 1407-1418.

Buson, L., Nardy, A., Muller, D., \& Chevrot, J.-P. (in press). The sociolinguistic repe- tition task : A new paradigm for exploring the cognitive coherence of language varieties.

Carrell, P. (1990). Rôle des schémas de contenu et des schémas formels. Acquisition et utilisation d'une langue étrangère. L'approche cognitive. Paris, Hachette, 16-29.

Compernolle, R. A. v. (2013). Concept appropriation and the emergence of 12 socios- tylistic variation. Language Teaching Research.

Conseil De L'Europe. (2001). Cadre européen commun de référence pour les langues : Apprendre, enseigner, Évaluer. Didier. Consulté sur https://rm.coe.int/16802fc3a8

Cuet, C. (2013). Acquisition du français par les Chinois. Recherches en didactique des langues et des cultures, 10(1), 2013, mis en ligne le 07 avril 2017, URL http://journals.openedition.org/rdlc/1532.

Dewaele, J.-M. (2002). Using socio-stylistics variants in advanced french interlan- guage : the case of "nous"/ "on". (S. Foster-Cohen, T. Ruthenberg, \& M. Po- schen, Eds.). John Benjamins.

Dewaele, J.-M. (2004). Retention or omission of the 'ne' in advanced french interlan- guage : the variable efect of extralinguistic factors. Journl of Sociolinguistics, 8(3), 433-450.

Dewaele, J.-M., \& Regan, V. (2002). Maîtriser la norme sociolinguistique en in- terlangue française : le cas de l'omission variable de 'ne'. Journal of French Language Studies, 12(2), 123-148.

Dewaele, J.-M., \& Wourm, N. (2002). L'acquisition de la compétence sociopragma- tique en langue étrangère. Revue Française de sociolinguistique appliquée, VII, 139-153.

Gautier, R. (2016). Développement des réseaux personnels et de la compétence socio- linguistique lors de séjours d'étude en france d'apprenants de fle américains et chinois (Thèse de doctorat non publiée). Université Grenoble Alpes. 
Howard, M. (2012). The advanced learner's sociolinguistic profile : On issues of individual differences, second language exposure conditions, and type of socio- linguistic variable. The Modern Language Journal, 96(1), 20-33.

Johnson, P. (1981). Effects on reading comprehension of language complexity and cultural background of a text. TESOL quarterly, 15(2), 169-181.

Kasper, G. (2001). Four perspectives on 12 pragmatic development. Applied linguis- tics, 22(4), 502530.

Lyster, R. (1994). The effect of functional-analytic teaching on aspects of french immersion students' sociolinguistic competence. Applied linguistics, 15(3), 263- 287.

Mougeon, R., Nadasdi, T., \& Rehner, K. (2002). État de la recherche sur l'appro- priation de la variation par les apprenants avancés du FL2 ou FLE. Acquisition et interaction en langue étrangère(17), 7-50.

Nardy, A., Chevrot, J., \& Barby, S. (2013). The acquisition of sociolinguistic varia- tion : looking back and thinking ahead. Linguistics, 2(51), 255-284.

Ranney, S. (1992). Learning a new script : An exploration of sociolinguistic compe- tence. Applied Linguistics, 13(1), 25-50.

Regan, V. (1995). The acquisition of sociolinguistic native norms. In B. Freed (Ed.), Second langugae acquisition in a study abroad context (p. 245-267). Jonh Benjamins.

Regan, V. (1996). Variation in french interlanguage : a longitudinal study of so- ciolinguistics competence. In R. Bayley \& D. Preston (Eds.), Second langugae acquisition in a study abroad context (p. 177-201). Jonh Benjamins.

Regan, V. (1997). Les apprenants avancés, la lexicalisation et l'acquisition de la compétence sociolingistique : une approche variationniste. AILE Acquisition et interaction en langue étrangère(9), 193-210.

Rumelhart, D. (1980). Schemata : The building blocks of cognition. In R. Spiro, B. Bruce, \& W. Brewer (Eds.), Theoretical issues in reading comprehension. Hillsdale, NJ : Lawrence Erlbaum Associates. 


\section{Annexes}

Tableau 2. Format des sections et sous-sections.

\begin{tabular}{|c|c|c|c|}
\hline Référence & Enoncé & Niveau & Variable \\
\hline FH1 & $\begin{array}{l}\text { Nous ne connaissions même } \\
\text { pas le président d(e) cette } \\
\text { association. }\end{array}$ & $\begin{array}{l}\text { Morpho } \\
\text { logique }\end{array}$ & $\begin{array}{l}\mathrm{Ne} \text { de } \\
\text { négation } \\
\text { présent }\end{array}$ \\
\hline $\mathrm{FH} 2$ & $\begin{array}{l}\text { Je m'interroge sur la façon } \\
\text { donT ils conçoivent } \\
\text { l'enseignement en général. }\end{array}$ & syntaxe & $\begin{array}{l}\text { Pronom } \\
\text { relatif dont }\end{array}$ \\
\hline FH3 & $\begin{array}{l}\text { C'esT un pays accueillant où il } \\
\text { ferait certes bon vivre. }\end{array}$ & $\begin{array}{l}\text { phonolo } \\
\text { gique }\end{array}$ & $\begin{array}{l}\text { liaison } \\
\text { facultative } \\
\text { réalisée } \\
\text { après } \\
\text { "c'est" }\end{array}$ \\
\hline FH4 & $\begin{array}{l}\text { Etant donné qu'il travaille à } \\
\text { l'hôpital, démissionner esT une } \\
\text { décision délicate. }\end{array}$ & $\begin{array}{l}\text { phonolo } \\
\text { gique }\end{array}$ & $\begin{array}{c}/ 1 / \\
\text { maintenu }\end{array}$ \\
\hline FH5 & $\begin{array}{l}\text { De plus, euh non, certains } \\
\text { articles évoquaient plutôt une } \\
\text { restructuration. }\end{array}$ & $\begin{array}{l}\text { pragmat } \\
\text { ique }\end{array}$ & $\begin{array}{l}\text { Particule } \\
\text { discursive } \\
\text { formelle }\end{array}$ \\
\hline FH6 & $\begin{array}{l}\text { Oui bon, la situation est certes } \\
\text { éminemment complexe. }\end{array}$ & $\begin{array}{l}\text { pragmat } \\
\text { ique }\end{array}$ & $\begin{array}{l}\text { Particule } \\
\text { discursive } \\
\text { formelle }\end{array}$ \\
\hline IH1 & $\begin{array}{l}\text { Tu vas pas mett(re) tes pompes } \\
\text { dégueu dans ma caisse, nan? }\end{array}$ & syntaxe & $\begin{array}{l}\text { Omission } \\
\text { de la } \\
\text { particule de } \\
\text { négation }\end{array}$ \\
\hline $\mathrm{IH} 2$ & $\begin{array}{l}\text { Nan c(e) que } \mathrm{j}(\mathrm{e}) \text { te parlais } \\
\mathrm{t}(\mathrm{ou}) \mathrm{t} \text { à l'heure, c'(é)tait un } \\
\text { aut(re) plan foireux. }\end{array}$ & syntaxe & $\begin{array}{l}\text { Pronom } \\
\text { relatif que } \\
\text { en lieu de } \\
\text { dont }\end{array}$ \\
\hline $\mathrm{IH} 3$ & $\begin{array}{l}\text { C'est un appart avec un pote } \\
\text { (il) y a pas la télé. }\end{array}$ & $\begin{array}{l}\text { phonolo } \\
\text { gique }\end{array}$ & $\begin{array}{l}\text { liaison } \\
\text { facultative } \\
\text { après } \\
\text { "c'est" non } \\
\text { réalisée }\end{array}$ \\
\hline IH4 & $\begin{array}{l}\text { Alors } \mathbf{i}(\mathbf{l}) \text { regarde le truc et } \mathbf{i}(\mathbf{l}) \\
\text { dit oh mais c'est quoi ça ?! }\end{array}$ & $\begin{array}{l}\text { phonolo } \\
\text { gique }\end{array}$ & $\begin{array}{l}\text { /1/ non } \\
\text { maintenu }\end{array}$ \\
\hline
\end{tabular}




\begin{tabular}{|c|c|c|c|}
\hline IH5 & $\begin{array}{l}\text { Écoute } t(u) \text { es vachement } \\
\text { sympa merci hein. }\end{array}$ & $\begin{array}{l}\text { pragmat } \\
\text { ique }\end{array}$ & $\begin{array}{l}\text { Particule } \\
\text { discursive } \\
\text { informelle }\end{array}$ \\
\hline IH6 & $\begin{array}{l}\text { Oh mais lui pour s'en } \\
\text { remett(re) franch(e)ment ça été } \\
\text { chaud. }\end{array}$ & $\begin{array}{l}\text { pragmat } \\
\text { ique }\end{array}$ & $\begin{array}{l}\text { Particule } \\
\text { discursive } \\
\text { informelle }\end{array}$ \\
\hline FnH1 & $\begin{array}{l}\text { C'esT un prix symbolique } \\
\text { même si le gouvernement veut } \\
\text { pas parler de gratuité. }\end{array}$ & $\begin{array}{l}\text { Morpho } \\
\text { logique }\end{array}$ & $\begin{array}{l}\text { Omission } \\
\text { de la } \\
\text { particule } \\
\text { de } \\
\text { négation }\end{array}$ \\
\hline $\mathrm{FnH} 2$ & $\begin{array}{l}\text { L'écueil majeur concerne la } \\
\text { manière qu'on enseigne } \\
\text { l'informatique à l'école. }\end{array}$ & syntaxe & $\begin{array}{l}\text { Pronom } \\
\text { relatif que } \\
\text { en lieu de } \\
\quad \text { dont }\end{array}$ \\
\hline $\mathrm{FnH} 3$ & $\begin{array}{l}\text { Certes les débordements dans } \\
\text { l'hémicycle étaient inévitables. }\end{array}$ & $\begin{array}{l}\text { phonolo } \\
\text { gique }\end{array}$ & $\begin{array}{l}\text { liaison } \\
\text { facultativ } \\
\text { e après } \\
\text { "étaient" } \\
\text { non } \\
\text { réalisée }\end{array}$ \\
\hline FnH4 & $\begin{array}{l}\text { Pour subvenir à ses besoins, } \\
\text { bien entendu, i' faut d'autres } \\
\text { sources de revenus. }\end{array}$ & $\begin{array}{l}\text { phonolo } \\
\text { gique }\end{array}$ & $\begin{array}{l}\text { /1/ non } \\
\text { maintenu }\end{array}$ \\
\hline FnH5 & $\begin{array}{l}\text { Nous pouvons sans doute nous } \\
\text { y soustraire hein. }\end{array}$ & $\begin{array}{l}\text { pragmat } \\
\text { ique }\end{array}$ & $\begin{array}{l}\text { Particule } \\
\text { discursive } \\
\text { informelle }\end{array}$ \\
\hline FnH6 & $\begin{array}{l}\text { Oh mais aujourd'hui les gens } \\
\text { ne se marient plus à } 25 \text { ans à } \\
\text { moins d'y être contraints. }\end{array}$ & $\begin{array}{l}\text { pragmat } \\
\text { ique }\end{array}$ & $\begin{array}{l}\text { Particule } \\
\text { discursive } \\
\text { informelle }\end{array}$ \\
\hline InH1 & $\begin{array}{l}\text { Ouais bein le gars i(l) n'avait } \\
\text { pas franch(e)ment } 1(\mathrm{e}) \text { choix au } \\
\text { final. }\end{array}$ & $\begin{array}{l}\text { Morpho } \\
\text { logique }\end{array}$ & $\begin{array}{l}\text { Ne de } \\
\text { négation } \\
\text { présent }\end{array}$ \\
\hline $\mathrm{InH} 2$ & $\begin{array}{c}\text { Essaye de pas } \mathrm{m}(\mathrm{e}) \text { refiler } \mathrm{l}(\mathrm{e}) \\
\text { matos pourri dont } \mathrm{t}(\mathrm{u}) \text { as parlé } \\
\text { l'aut(re) fois. }\end{array}$ & syntaxe & $\begin{array}{l}\text { Pronom } \\
\text { relatif } \\
\text { dont }\end{array}$ \\
\hline InH3 & $\begin{array}{c}\text { C'esT un sale gosse j(e) te jure } \\
\text { il est grave. }\end{array}$ & $\begin{array}{l}\text { phonolo } \\
\text { gique }\end{array}$ & $\begin{array}{l}\text { liaison } \\
\text { facultativ } \\
\text { e réalisée } \\
\text { après } \\
\text { "c'est" }\end{array}$ \\
\hline
\end{tabular}




\begin{tabular}{|c|c|c|c|}
\hline InH4 & $\begin{array}{l}\text { Ouais il bosse tout } 1(\mathrm{e}) \text { temps } \\
\text { c'est abuser. }\end{array}$ & $\begin{array}{l}\text { phonolo } \\
\text { gique }\end{array}$ & $\begin{array}{c}/ 1 / \\
\text { maintenu }\end{array}$ \\
\hline InH5 & $\begin{array}{c}\text { Euh non en vrai j'avais bien les } \\
\text { boules de pas capter ses } \\
\text { questions. }\end{array}$ & $\begin{array}{l}\text { pragmat } \\
\text { ique }\end{array}$ & $\begin{array}{l}\text { Particule } \\
\text { discursive } \\
\text { formelle }\end{array}$ \\
\hline InH6 & $\begin{array}{l}\text { Oui bon en même temps } \\
\text { comment i(l) s(e) galère avec sa } \\
\text { meuf. }\end{array}$ & $\begin{array}{l}\text { pragmat } \\
\text { ique }\end{array}$ & $\begin{array}{l}\text { Particule } \\
\text { discursive } \\
\text { formelle }\end{array}$ \\
\hline
\end{tabular}

